\title{
Participatory Consciousness and Complicity
}

\author{
BRENT DAVIS, Co-Editor \\ University of British Columbia (Canada) \\ RENATA PHELPS, Co-Editor \\ Southern Cross University (Australia)
}

This is our final issue as co-editors of Complicity, and so we would like to begin our remarks with a brief expression of appreciation to members of the community who have assisted in preparing and reviewing manuscripts. In particular, we thank our Book Review Editor, Kristopher Wells, who always seemed to be a step ahead of us in the process. From the birth of Complicity following the first Complexity and Education conference in 2003 we have seen a steady increase in submissions and readership, reflecting the growth of interest in complexity theories by educators internationally. We wish the journal well and are delighted to see it move into the capable hands of a strong editorial team: Deborah Osberg (Editor-in-Chief), Bill Doll (Associate Editor), Donna Trueit (Associate Editor), and Darren Stanley (Book Review Editor).

As we considered topics for this final editorial piece, one issue kept pressing itself into our awarenesses-namely, the changing landscapes of possibility and what these might mean for education and educational research. For the most part, discussions of the topic seem to be organized around a complexivist sensibility (often implicitly) and emergent technologies (usually explicitly). ${ }^{1}$

As has been noted by many commentators, the word technology tends to be popularly understood in terms of physical tools and machines. Occasionally language, mathematics, and other areas of human competence are 
included, but the principal interest is usually the question of how we shape our technologies, as opposed to how we embody our technologies-that is, how choices for and manners of representation affect what we notice, know, do, and are. Stated somewhat differently, technologies participate in the transformation of minds-that is, experiences of consciousness-by shaping how experiences are represented. There is an abundance of evidence for this claim in the archeological record, in which there are indications of clear and rather abrupt advances in technologies that correspond with different social structures, different intellectual demands, and different preoccupations. These shifts might also be described as sudden lurches in intelligence as humans expanded their repertoires of possibility at a pace that simply cannot be explained through biological evolution.

Different commentators organize eras and developments in different ways. For the sake of brevity, we draw on just a single frame here-one developed by psychologist Merlin Donald. (Other accounts are cited in a endnote..$^{2}$ ) Donald argues that the complexity of technology can be taken as an indication of the level of consciousness. For instance, it appears that until about 2 million years ago, the prevailing technologies were tools that were found rather than made, such as sticks and broken rocks. These items were for immediate use by individuals. They are the sorts of things that call on only short-term memory, and so are discarded and perhaps forgotten once the task is complete. Donald suggests such technologies are indicative of an episodic consciousness - an awareness of the here and now, but of little more.

According to current interpretations of the archeological record, a new category of technology appeared about two million years ago, one that was associated with the deliberate manufacturing of tools. Such tools required long-term memories that made it possible to select from past experience and contemplate future needs. Using the phrase mimetic consciousness, Donald suggests such a mode of awareness was more social and highly reliant on imitation (mimesis).

According to Donald, as these technologies proliferated and were improved, there arose a need for more flexible technologies to collect and organize ideas. Donald associates this transition with the emergence of sophisticated languaging technologies that made it possible to distribute memory across communities and to extend capacities to interpret the past and project into the future. This mythic consciousness, he suggests, emerged a few hundred thousand years ago, and is marked by the appearance of more abstract, less pragmatic artifacts.

Much more recently, in the order of 5,000-10,000 years ago, capacities to think abstractly were greatly enhanced. Donald associates this sort of theoretical consciousness with the technology of writing, which makes it 
possible to deal with information in a more detached way and to stabilize insights by offloading memory onto physical artifacts.

Donald ends his analysis there, but it is interesting to extrapolate his thinking. Using the markers he suggests-that is, enhanced technologies, improved abilities to mass-link minds, increased intellectual capacities that cannot be explained by biological evolution, emergence of new memory systems to store and access information, and shifts in social and cultural organization - it may be that we are on the cusp of a new mode of consciousness. In fact, projects are underway to digitally chronicle every aspect of a person's life, enabled by advances in video and data storage technologies. Clearly such enhanced memories present the possibility of dramatically transforming experience-and, hence, consciousness. It is probably premature to try to specify the nature of a new form of consciousness that might be triggered and enabled by new technologies. However, an emergent mode of being will likely embody growing awareness of the complexity of learning phenomena and the participatory nature of knowing-where we follow Jenkins and colleagues ${ }^{3}$ in their description of "participatory culture":

A participatory culture is a culture with relatively low barriers to artistic expression and civic engagement, strong support for creating and sharing one's creations, and some type of informal mentorship whereby what is known by the most experienced is passed along to novices. A participatory culture is also one in which members believe their contributions matter, and feel some degree of social connection with one another (at the least they care what other people think about what they have created).

Working from this frame, it seems reasonably to argue that education, educational research, and academic publishing can, and perhaps should be profoundly participatory-wholly complicit in the emergence of new possibilities. We are participating in the evolutions that we are witnessing. Moreover, complexity thinking has helped to foreground the collective nature of evolving possibilities. Current transformations and emergent forms involve many minds and mediating technologies that, in turn, affect not only personal imaginations, motivations, and consciousnesses, but also cultural senses of truth, rationality, and justification. Against this backdrop, it is perhaps ironic that much of educational practice and research activity is still organized around the linearity of print-based texts, the singular authority of a mandated curriculum, and an ideal of individualism (embodied in evaluation schemes, classroom arrangements, teaching strategies, and the "great man" syndrome in the research literature). The irony increases when one considers that the massive successes of video-gaming and related technologies are at least partly due to the ways their creators have deliberately exploited principles of learning related to collectivity, sociocultural contexts, shifting identities, embodiment, non-linear and emergent pathways, implicit 
associations, effortful study, and other notions that are explicitly informed by complexity thinking.

The issues here could be phrased in terms of the way that "teaching" emerged and evolved alongside human consciousness. For instance, with the here- and now-ness of Donald's episodic consciousness, teaching would be largely accidental and nondeliberate, as actors copy the actions of others or respond to their changing contexts and environments. With the development of mimetic consciousness, and as humans created task-specific tools, teaching had to become more intentional, taking on dimensions of showing and correcting.

With the emergence of abstract language and sophisticated narratives of a mythic consciousness, teaching would have had to be transformed and elaborated once again as the needs to rehearse and interpret were added to established instructional practices of showing, correcting, and modeling. Correspondingly, the emergence of a theoretical consciousness prompted an explosion of possible roles, presenting a need for a mode of teaching that is as much about anticipation of the future as maintenance of established knowledge.

It is becoming more obvious that education is nearing a new crossroads as it faces another shift in emphasis, away from individuals who pass on established knowledge and toward collectives who elaborate emergent knowings. Teaching and educational research are taking on a more participatory emphasis, and what they might become will have everything to do with the forms that are created for and by learning.

This issue of forms is prominently represented in each of the feature articles in this issue of Complicity. In the opening essay, Claudia Ruitenberg looks to cartography as a mode of representation that, she argues, is well suited capturing the complexity of aspects of the educational project. In the next pair of articles, Tyler Volk and Jeff Bloom develop the assertion of metapatterns - that is, iteratively produced patterns or principles that are common to a large set of systems - are useful for formulating new questions for educational research. In the subsequent article, Lyubov Laroche, Cynthia Nicol, and Jolie-Mayer-Smith look to another set of forms-including chaotic attractors and fuzzy boundaires-as a means to make sense of the pragmatic responsibilities of science educators. And in the final of the four feature articles in this issue, Jeanne Adele Kentel and Douglas Karrow interrogate the space of technocratic approaches to teacher education as they seek ways of engaging the bodymind in order to bring about a broader, critical educative focus in teacher education.

In the Semantic Play section of this issue, we are thrilled to begin with an invited contribution from Ian Stewart. It was in a book that Ian co-authored with Jack Cohen, The Collapse of Chaos, ${ }^{4}$ that we found our inspiration for 
the title of this journal. It seems only fitting to revisit the thinking that first contributed to this bit of sematic play. Also featured in this issue are George Siemens who discussions connecting, Stewart Hase and Chris Kenyon who take on heutagogy, and Noel Gough who elaborates Deleuze and Guattari's rhizomatics in his take on rhizosemiotics.

The issue ends with several book reviews, collected and introduced by Kristopher Wells. Kris also takes the opportunity to introduce the new Book Review Editor, Darren Stanley.

We close by expressing once again our appreciation to members of the Complicity journal for their assistance and support over the past four years. It's been fun.

\section{Endnotes}

1. This discussion is based in large part on "Chapter 8: Learning Forms," from B. Davis, D. Sumara, and R. Luce-Kapler, Engaging minds: Changing teaching in complex times (New York: Routledge, 2008).

2. The account of the emergence of consciousness that is provided here is based on Merlin Donald, Origins of the modern mind: Three stages in the evolution of culture and cognition (Cambridge, MA: Harvard University Press, 1991). Other accounts include Terrance Deacon, The symbolic species: The co-evolution of language and the brain (New York: W.W. Norton, 1997); Daniel C. Dennett, Kinds of minds: Towards an understanding of consciousness (New York: Basic, 1997); Julian Jaynes, The origin of consciousness and the breakdown of the bicameral mind (New York: Penguin, 1979); Steven Mithen, The prehistory of the mind: The cognitive origins of art, religion and science (London: Thames and Hudson, 1996).

3. On page 3 of H. Jenkins, K. Clinton, R. Purushotma, A.J. Robison, \& M. Weigel, Confronting the challenges of participatory culture: Media education for the 21st century (Chicago: The MacArthur Foundation, 2007). Downloaded on May 14 from http:/ / www.digitallearning.macfound.org/atf/cf/\%7B7E45C7E0-A3E04B89-AC9C-E807E1B0AE4E\%7D/JENKINS_WHITE_PAPER.PDF

4. I. Stewart and J. Cohen, The collapse of chaos: Discovering simplicity in a complex world (New YOrk: Penguin, 1994).

(C) Copyright 2007. The authors, Brent Davis and Renata Phelps, assigns to the University of Alberta and other educational and non-profit institutions a non-exclusive license to use this document for personal use and in courses of instruction provided that the article is used in full and this copyright statement is reproduced. The authors also grant a non-exclusive license to the University of Alberta to publish this document in full on the World Wide Web, and for the document to be published on mirrors on the World Wide Web. Any other usage is prohibited without the express permission of the authors. 
